\title{
Numerical study on mitigating tsunami force on bridges by an SPH model
}

\author{
Zhangping Wei ${ }^{1} \cdot$ Robert A. Dalrymple ${ }^{1}$
}

Received: 13 October 2015 / Accepted: 15 April 2016 / Published online: 12 May 2016

(C) Springer International Publishing Switzerland 2016

\begin{abstract}
This study applies the numerical model of GPUSPH, an implementation of the weakly compressible Smoothed Particle Hydrodynamics method on graphics processing units, to investigate tsunami forces on bridge superstructures and tsunami mitigation on bridges by using a service road bridge and an offshore breakwater. The capability of GPUSPH to predict tsunami forces on bridges is first validated by simulating a laboratory experiment on tsunami impacting a bridge with different configurations of superstructures. To address the uncertainty of tsunami generation with the gate falling method used in the laboratory, this study proposes a new tsunami wave generation method that makes use of the laboratory free-surface measurements to replicate the wave. Furthermore, the tsunami force, in particular, the first impact force, on bridges is reasonably predicted by GPUSPH. Next additional numerical experiments built upon the laboratory work are carried out to examine the efficiency of tsunami mitigation by an upwave service road bridge and an offshore breakwater. It is found that a two-girder service road bridge is effective in reducing tsunami forces on the main bridge. Furthermore, a breakwater can also reduce tsunami forces on a bridge, and there is an optimal distance between the breakwater and the bridge to achieve the best reduction effect. However, the tsunami mitigation structures experience a strong tsunami force, which may lead to the failure of these structures.
\end{abstract}

Zhangping Wei

zwei@jhu.edu; zwei.coast@gmail.com

Robert A. Dalrymple

rad@jhu.edu

1 Department of Civil Engineering, Johns Hopkins University, Baltimore, MD 21218, USA
Keywords Tsunami · Bridge · Wave-structure interaction · Hydrodynamic force $\cdot$ Hazard mitigation $\cdot$ Smoothed Particle Hydrodynamics

\section{Introduction}

Several major tsunamis in the past decades have caused significant damages to bridge structures. Post-disaster surveys have found that scouring of bridge foundations, loss of abutments, uplift due to buoyancy, and bridge superstructure sliding are the major causes of a bridge failure during a tsunami (e.g., Saatcioglu et al. 2006; Kawashima and Buckle 2013). All these failure modes indicate that a bridge failure is closely related to the hydrodynamics of a tsunami, in particular, tsunami forces on bridges. Moreover, countermeasures for mitigating the effect of tsunami forces on bridges should be studied and further implemented in engineering practices. To address the above issues, this work investigates tsunami forces on bridge superstructures, and examines the efficiency of measures used in practice for mitigating tsunami forcing on bridges.

In addition to field surveys, laboratory experiments and numerical simulations have been conducted to study tsunami impact on bridges. A number of experimental studies were carried out after the 2004 Indian Ocean Tsunami, and this study briefly reviews some of them. Arnason et al. (2009) conducted a set of flume experiments on a tsunami impacting vertical columns that resemble bridge piers in real life. Their results show that tsunami forces are influenced by the shapes and orientations of bridge piers. Kosa et al. (2010) investigated tsunami forces on bridge girders by considering the impact of breaking waves and non-breaking waves. They observed that for two waves with approximately the same height, the breaking wave gives a higher horizontal force, 
while the non-breaking wave results in a larger uplift force. Lau et al. (2011) examined tsunami forces on bridge decks, and further categorized them into four types, i.e., impulsive, slowly varying, uplift, and additional gravity forces. Furthermore, a few experiments also examined tsunami forces on multiple components of a bridge (e.g., Nakao et al. 2013), and even a whole bridge model (e.g., Iemura et al. 2007).

With the rapid development of computer hardware and numerical methods, numerical models are also used to simulate these forces. To mention some of them, Lau et al. (2011) used Flow-3D ${ }^{\circledR}$ to simulate tsunami forces on a bridge deck involving wave impact and overtopping, and they obtained a good agreement with the experimental data for both hydrodynamic pressure and wave force. Hayatdavoodi et al. (2014) applied a two-phase flow solver InterFoam to investigate the force of a tsunami-like solitary wave on bridge deck and girders. They not only compared the experimental force data, but also explored the entrapped air pockets impact on force predictions. Recently, we have applied the GPU-accelerated Smoothed Particle Hydrodynamics (SPH) model, GPUSPH, to investigate tsunami forces on bridge piers, showing that GPUSPH is able to accurately predict tsunami forces on different shapes of bridge piers (Wei et al. 2015).

Furthermore, mitigation of tsunamis was also addressed in several works. For example, coastal structures, such as seawalls and breakwaters, serve as major structural countermeasures to mitigate tsunamis (see e.g., Fujima 2006; Thomas and Cox 2011). Recently, the so-called environment-friendly countermeasures for attenuating tsunamis gain increasing amount of attentions (see e.g., Kathiresan and Rajendran 2005; Tanaka et al. 2007). It is seen that the above countermeasures are proposed to mitigate tsunamis impact on coastal area in a general way, and a very few works have investigated specific countermeasures to mitigate tsunami impact on a bridge (Iemura et al. 2007; Nakao et al. 2013). Considering the massive loss of bridges caused by tsunamis, more effort should be devoted to study mitigating tsunami forcing on bridges. This paper is going to present some preliminary work on this topic by using the numerical model of GPUSPH (Hérault et al. 2010). The model is first validated by the laboratory experiment of Nakao et al. (2013) on tsunami forcing on bridge superstructures, and then additional numerical experiments are conducted to investigate tsunami force mitigation on bridges. The rest of the paper is organized as follows. Section 2 briefly reviews the fundamentals of the numerical model. Section 3 introduces the physical experiment and its corresponding numerical setup. Then the main work in this paper is divided into two parts. The first part validates the capability of GPUSPH to predict tsunami forcing on different bridge superstructures in Sects. 4 and 5. The second part, which is an extension of the first part, examines the efficiency of man-made structures such as a service road bridge and a breakwater for mitigating tsunami forces on bridges in Sect. 6. Finally, conclusions are made in Sect. 7.

\section{Numerical model}

\subsection{GPUSPH}

This study uses the open-source fluid-dynamics SPH code: GPUSPH $^{1}$, which is an implementation of the weakly compressible SPH (WCSPH) method on graphics processing units (Hérault et al. 2010); the basic equations of GPUSPH follow an early version of SPH code: SPHysics (e.g., GomezGesteira et al. 2012). The theoretical formulations of the WCSPH method and its numerical implementations could be found in many references (e.g., Monaghan 1992, 1994; Dalrymple and Rogers 2006). However, for the completeness of this work, the fundamentals of the SPH method are briefly reviewed and the governing equations of the WCSPH method are presented in this section.

In an SPH model, the computation domain is discretized into particles, which carry material properties such as mass, velocity, density, and pressure. A function $f(\mathbf{r})$ of the particle position vector $\mathbf{r}$ is approximated by the following integral (Monaghan 1992):

$f(\mathbf{r})=\int f\left(\mathbf{r}^{\prime}\right) W\left(\mathbf{r}-\mathbf{r}^{\prime}, h_{s}\right) \mathrm{d} \mathbf{r}^{\prime}$,

where $h_{s}$ is the smoothing length defining the influence area of the weighting function $W$. In this study, a quintic function of Wendland (1995) is used:

$W(q)=\alpha_{D}\left(1-\frac{q}{2}\right)^{4}(1+2 q) ; \quad 0 \leq q \leq 2$,

where $q=\frac{\left|\mathbf{r}_{i}-\mathbf{r}_{j}\right|}{h_{s}}$ is the non-dimensional distance between particles $i$ and $j$; and $\alpha_{D}=21 /\left(16 \pi h_{s}^{3}\right)$ is used.

In a discrete notation, Eq. (1) can be further written as

$\left\langle f\left(\mathbf{r}_{i}\right)\right\rangle=\sum_{j} \frac{m_{j}}{\rho_{j}} f\left(\mathbf{r}_{j}\right) W\left(\left|\mathbf{r}_{i}-\mathbf{r}_{j}\right|, h_{s}\right)$,

where $i$ is the particle of interest; $\left\langle f\left(\mathbf{r}_{i}\right)\right\rangle$ is the approximation for $f(\mathbf{r})$ at $\mathbf{r}_{i} ; j$ is the particle within a radius of $2 h_{s}$ of the particle $i$; and $m$ is the particle mass.

Furthermore, the first-order and second-order spatial derivatives (e.g., gradient, divergence, and Laplacian) of the function $f(\mathbf{r})$ are obtained by applying the corresponding operators on the discretized $f(\mathbf{r})$ in Eq. (3) (see e.g., Monaghan 1992; Lo and Shao 2002). Then the differential

\footnotetext{
1 The code is freely available at: http://www.gpusph.org.
} 
operators are applied to discretize the Navier-Stokes equations:

$$
\begin{aligned}
& \frac{\mathrm{D} \rho}{\mathrm{D} t}=-\rho \nabla \cdot \mathbf{u} \\
& \frac{\mathrm{D} \mathbf{u}}{\mathrm{D} t}=-\frac{\nabla P}{\rho}+\mathbf{g}+v_{0} \nabla^{2} \mathbf{u}+\frac{1}{\rho} \nabla \cdot \tau,
\end{aligned}
$$

where $t$ is the time; $\rho$ is the fluid density; $\mathbf{u}$ is the particle velocity; $P$ is the pressure; $g$ is the gravitational acceleration; $\nu_{0}$ is the laminar kinematic viscosity; and $\tau$ is the turbulence stress tensor, which is approximated by the sub-particle scale model of Dalrymple and Rogers (2006).

As the fluid is assumed to be weakly compressible in this study, the pressure in Eq. (5) is directly computed by using the equation of state (Monaghan 1992) by

$P=\beta\left[\left(\frac{\rho}{\rho_{0}}\right)^{\gamma}-1\right]$,

where $\rho_{0}$ is the initial density; $\gamma$ is chosen to be 7; and the parameter $\beta$ is calculated by

$\beta=\frac{\rho_{0} C_{s 0}^{2}}{\gamma}$,

where $C_{s 0}$ is the speed of sound evaluated with $\rho=\rho_{0}$. The real speed of sound limits the numerical time step to be very small. To address this issue, Monaghan (1994) proposed to use a reduced speed of sound in numerical simulation. However, the adjusted speed of sound should satisfy $C_{s 0} / u_{\max }>10$ (where $u_{\max }$ is the maximum velocity in the simulation) to avoid unphysical density fluctuation. Furthermore, the Shephard filter is performed every 20 steps to smooth the density field (Dalrymple and Rogers 2006).

In an SPH model, a numerical boundary condition plays an important role in obtaining correct numerical results. As an SPH particle approaches a wall boundary, its kernel does not have full support domain any more. To address this issue,
Dalrymple and Knio (2000) proposed to represent the wall boundary with several layers of dynamic boundary particles, so that the particle of interest inside the computation domain will have a full kernel support near the wall boundary. The smoothing length $h_{\mathrm{S}}$ is chosen to be 1.3 times of the particle size $\Delta p$ in this study, and then only three layers of dynamic boundary particles are needed. These dynamic boundary particles share the same equations of continuity and state as the fluid particles placed inside the domain; however, their positions and velocities remain unchanged in time. In this study, rigid boundaries include bridge structures (e.g., piers, girders, and decks) are represented by dynamic boundary particles, which are also used to measure the hydrodynamic force on structures. Basically, for each dynamic boundary particle, the force exerted by its neighboring fluid particles is first obtained, and then the total hydrodynamic force on the structure is the summation of forces on individual dynamic boundary particles.

\subsection{Numerical model validation}

The primary focus of this work is to predict the tsunami hydrodynamic force on bridge structures by using the SPH method. In Wei et al. (2015), GPUSPH was applied to simulate an experiment on tsunami impacting individual bridge piers. By fully replicating the tsunami generation process in the laboratory, GPUSPH accurately reproduces the hydrodynamic force on bridge piers. In this study, we further conduct a numerical experiment to verify its capability on force prediction by comparing with an analytical solution. The numerical experiment setup is shown in Fig. 1a. Considering a water tank has a water depth of $h$, and a width of $b$, the hydrostatic force exerted on its vertical wall is given by

$F_{\mathrm{s}}=\frac{1}{2} \rho g b h^{2}$

In the numerical model, the tank width is fixed to be $0.5 \mathrm{~m}$, but several water depths are tested. The domain is discretized
Fig. 1 a Sketch of the numerical experiment on hydrostatic force exerting on a vertical wall, and $\mathbf{b}$ comparison of analytical solution and numerical results of the hydrostatic force. Water depths $0.25,0.5,0.75,1,1.25,1.5$, and $1.75 \mathrm{~m}$ are simulated by GPUSPH
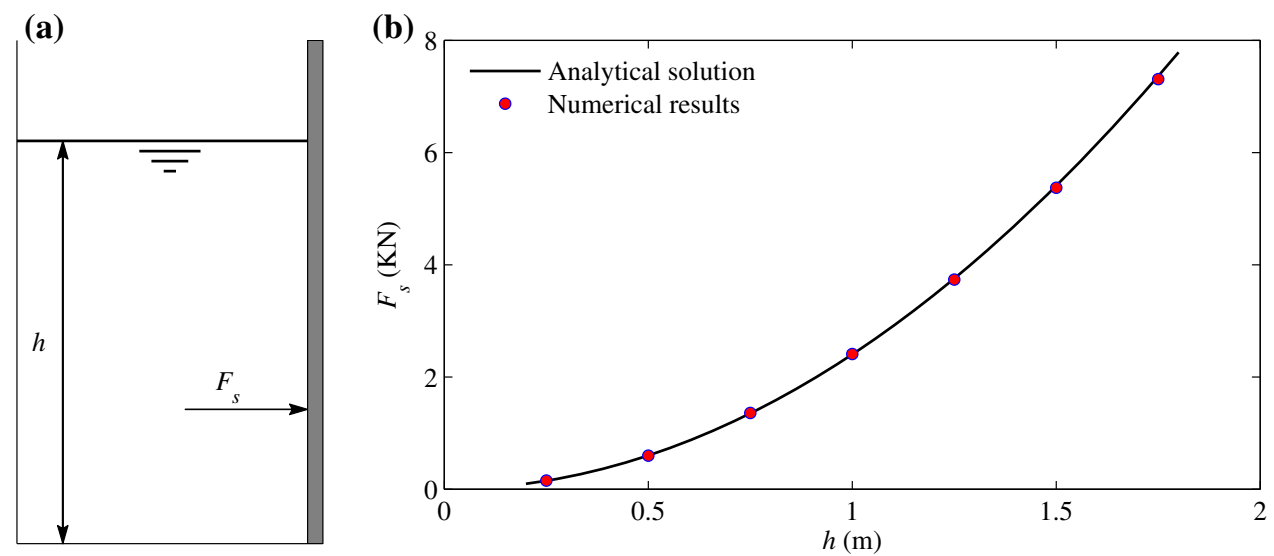
with a particle size of $\Delta p=0.005 \mathrm{~m}$, and the dynamic boundary condition is used for wall boundaries. The hydrostatic force on the vertical wall is obtained by summarizing hydrodynamic forces on individual dynamic boundary particles representing that wall. In reality the water is still, but a fluctuating force was observed in the simulation because of the weakly compressible nature of the SPH method used in this study. To address this issue, the simulation was run long enough to obtain a stable force profile, and then a movingaveraging filter was used to remove the acoustic noises from the computed hydrostatic force, resulting in a mean value. The difference between the output hydrostatic force from GPUSPH (i.e., the one with the acoustic noises) and the filtered mean force is evaluated by the coefficient of variation of the root-mean-square error (RMSE) (see e.g., Wei et al. 2015). It is seen that the coefficient of variation of RMSE is within $5 \%$, indicating that it is quantitatively acceptable to use the filtered mean force to represent the computed hydrostatic force on the vertical wall. Figure $1 b$ shows the comparison of the computed hydrostatic force with the analytical solution of Eq. (8). It is seen that numerical results match with the analytical solution well, and this good agreement further justifies the capability of GPUSPH on force prediction.

\section{Physical experiment and numerical model setup}

\subsection{Laboratory experiment}

Nakao et al. (2013) reported a series of flume tests conducted by Public Works Research Institute of Japan to study the bridge behavior subjected to the tsunami-induced force. To investigate tsunami velocity up to $8 \mathrm{~m} / \mathrm{s}$ impacting bridge structures as observed during the 2011 Great East Japan Tsunami, the laboratory experiment scale was determined to be $1 / 20$, and a sketch of the experimental setup is shown in Fig. 2a. The flume is $30 \mathrm{~m}$ long, $1.0 \mathrm{~m}$ wide, and $1 \mathrm{~m}$ deep, and a gate located at $x=12 \mathrm{~m}$ divides the flume into two sections. The tsunami in the laboratory was generated by releasing the gate, which freely falls forward into the flume. It should be pointed out that the gate falling process is not
Fig. 2 a Sketch of the physical experimental setup of Nakao et al. (2013), b sketch of the numerical model setup of the experiment of Nakao et al. (2013) in GPUSPH, c 3D view of Type I bridge with a single deck, $\mathbf{d}$ top view of Type I bridge, e $3 D$ view of Type II bridge with a deck and four girders, and $\mathbf{f}$ top view of Type II bridge
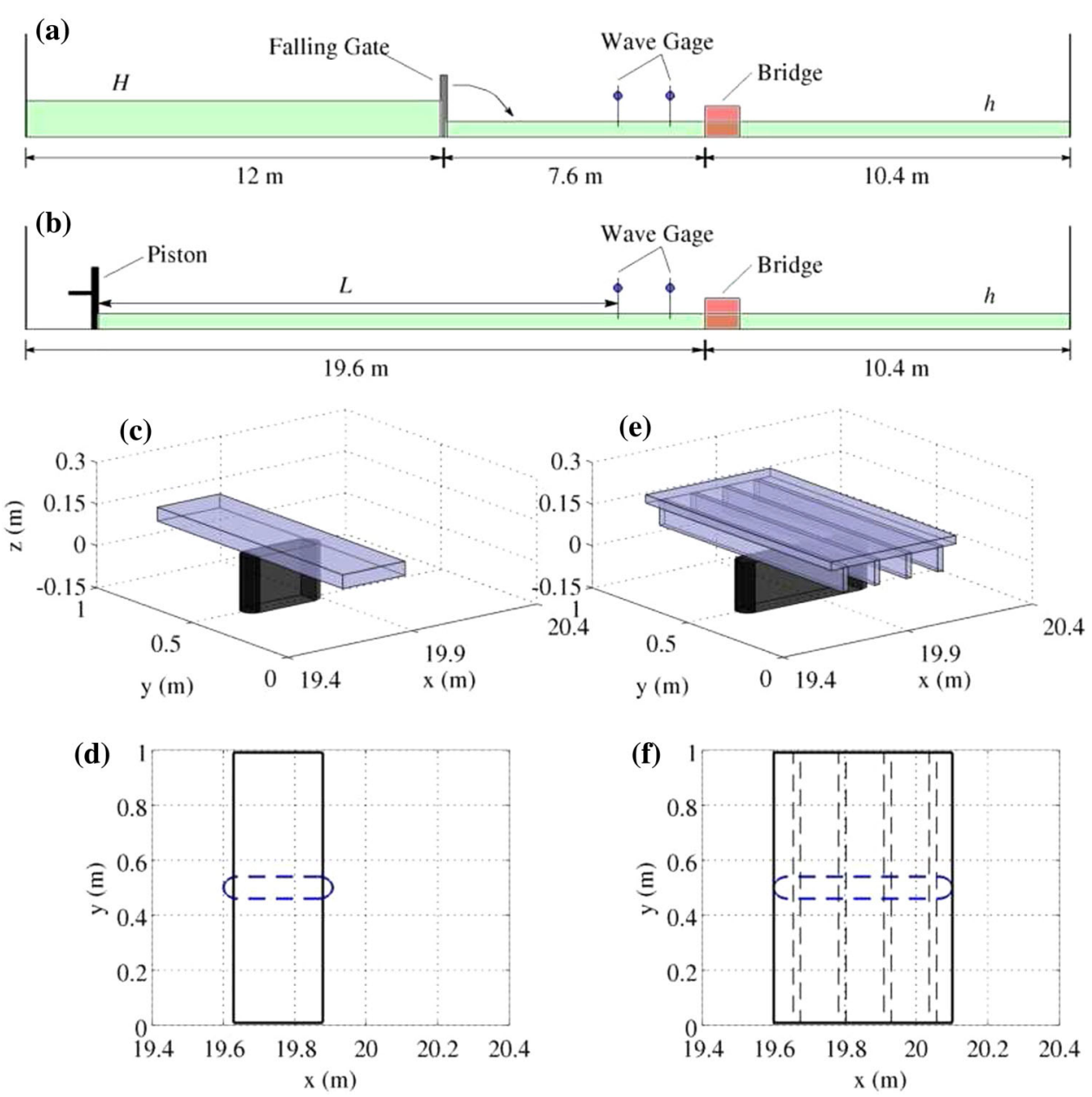
only influenced by the initial hydrostatic forces at its two sides, but also the mass of the gate, the hinge, and the timevarying hydrodynamic loadings during the falling process. As a result, it is very challenging for a numerical model to accurately replicate the whole process, and this issue will be further addressed in next section. The reservoir has a water depth of $H$, and the downstream flume has a water depth of $h$. Although different combinations of $H$ and $h$ were used in the laboratory, this work only simulates cases with $h=0.15 \mathrm{~m}$ and a tsunami bore height of $A=0.1 \mathrm{~m}$, as the corresponding experimental data are available to the authors through the US-Japan Tsunami Modeling Workshop held at Oregon State University in December 2014. Considering the physical experiment scale is $1 / 20$, the experiment actually resembled a 2-m-high tsunami propagation over a 3-m deep water in reality.

A bridge structure was placed nearshore, and the distance between the gate and the leading part of the bridge is about $7.6 \mathrm{~m}$. This study considers two bridge models, which are denoted as Type I and Type II bridges hereafter. The Type I bridge consists of a single deck sitting above a bridge pier, a $3 \mathrm{D}$ view and a top view of Type I bridge are shown in Fig. 2c, d, respectively. The dimension of the deck of Type I bridge is $0.25 \mathrm{~m}$ long in the longitudinal direction of the flume, $0.985 \mathrm{~m}$ wide in the transverse direction of the flume, and $0.05 \mathrm{~m}$ thick. The round-shaped pier of Type I bridge is $0.305 \mathrm{~m}$ long, $0.08 \mathrm{~m}$ wide, and $0.18 \mathrm{~m}$ high. There are two bearing supports with a dimension $(0.01 \times 0.04 \times 0.02$, $L \times W \times H$, unit m) connecting the pier and the deck, giving the bottom of the deck with a height of $0.2 \mathrm{~m}$ above the floor. For Type II bridge, its superstructure was made of a deck and four girders, which were supported by a bridge pier underneath. Similarly, a 3D view and a top view of Type II bridge are shown in Fig. 2e, f, respectively. The dimension of the deck of Type II bridge is $0.5 \mathrm{~m}$ long, $0.985 \mathrm{~m}$ wide, and $0.03 \mathrm{~m}$ thick. Each of the four girders of Type II bridge has a dimension of $0.02 \times 0.985 \times 0.07(L \times W \times H$, unit $\mathrm{m}$ ), and the distance between girders is about $0.107 \mathrm{~m}$. The pier of Type II bridge also has two round-shaped edges, and it is $0.5 \mathrm{~m}$ long, $0.08 \mathrm{~m}$ wide, and $0.18 \mathrm{~m}$ high. Similar to the setup in Type I bridge, four bearing supports were used to connect girders to the bridge pier in Type II bridge.

In terms of laboratory measurements, time-series freesurface elevations were measured by two wave gages (denoted as 1 and 2), which were located at 2.5 and $1 \mathrm{~m}$ offshore of the bridge structure, respectively. The primary concern of the experimental study was to examine tsunami forces on bridge superstructures, and therefore both horizontal and vertical tsunami hydrodynamic forces on bridge superstructures were measured by biaxial load cells located at bearing supports, which were used to connect the bridge pier and the bridge superstructure as mentioned above.

\subsection{Numerical model setup}

For the numerical model setup in GPUSPH, several modifications or adaptations are made with respect to the actual laboratory setup. First of all, as the flume and structures are discretized into a set of particles in the numerical model, tsunami forces on the bridge superstructure are obtained by summarizing the force exerted on individual particles that are used to represent the structure (Wei et al. 2015). Therefore, there is no need for the numerical model to include bearing supports in which biaxial load cells were installed in the laboratory, and then dimensions of the bridges are slightly adjusted. For Type I bridge in the numerical setup, the $0.18-\mathrm{m}$-high bridge pier is vertically extended by $0.02 \mathrm{~m}$ for considering the space occupied by the biaxial load cells in the laboratory; this modification does not change the dimension of the superstructure, i.e., the single deck. With respect to the 0.02 -m-high bearing supports in Type II bridge, they are considered as part of the girders in the numerical setup. Since the girder is about $0.985 \mathrm{~m}$ wide, this modification slightly increases the frontal area of girders.

Secondly, there is an uncertainty regarding the tsunami generation in the laboratory by the falling gate method, which is different from some well-controlled methods for generating tsunamis, such as dam-break (e.g., Arnason et al. 2009) that could be precisely reproduced in our previous work in Wei et al. (2015). When the falling gate method is tested by a numerical model, one has to try different gate falling profiles in the hope that the predicted time-series free-surface profiles would match the measured ones at the two wave gages. In this study, to avoid the tedious approach involving trial and error, an alternative wave generation method using a piston wavemaker is proposed, and the sketch of the numerical setup is shown in Fig. 2b. The basic idea of this method is to drive the piston wavemaker by using the measured timeseries free-surface profile at Gage 1 that is $2.5 \mathrm{~m}$ offshore of the bridge, and then we validate the predicted tsunami freesurface profile by using the measured data at Gage 2 that is $1 \mathrm{~m}$ offshore of the bridge. The following equation is used to convert the measured free-surface elevation $\eta(t)$ at Gage 1 to the piston wavemaker velocity $u_{\mathrm{p}}(t)$ :

$u_{\mathrm{p}}(t)=\frac{C \eta\left(t+t_{L}\right)}{h+\eta\left(t+t_{L}\right)}$,

where $C$ is an averaged wave celerity and it is defined by $C=$ $\sqrt{g(h+A)}$ with the tsunami bore height $A=\max (\eta(t))$. The variable $t_{L}$ is introduced to consider the amount of time needed for the wave propagating a distance $(L)$ between the piston location $x(t)$ and Gage 1, and it is given by

$t_{L}=\frac{L-x(t)}{C}$ 
It is noted that Eq. (9) represents the relationship between the shallow water wave celerity and its free-surface elevation. This relationship is widely used by mesh-based numerical models to generate a solitary wave at a boundary (see e.g., Wei and Jia 2013, 2014). Although the primary goal is to validate the free-surface profile at Gage 2, a shift of the piston wavemaker location could also allow to compare the freesurface profile at Gage 1, as shown in the following sections.

After the above adaptations, the experimental domain is discretized into a collection of particles with a fixed particle size. To evaluate the model convergence, three particle sizes $(\Delta p=0.01,0.0075$ and $0.005 \mathrm{~m})$ are used by GPUSPH. It is noted that $\Delta p=0.005 \mathrm{~m}$ was previously used in Wei et al. (2015) to simulate a laboratory experiment with a similar dimension of this work, and it was able to resolve water drops due to tsunami-pier impact. With $\Delta p=0.005 \mathrm{~m}$, the total number of particles for Type I bridge is about 20 million, of which 15 million are fluid particles. The remaining particles are used to represent the boundary and the piston wavemaker. For Type II bridge, there is about 22 million particles, of which 16 million are fluid particles. This study utilizes the multi-GPU version of GPUSPH (Rustico et al. 2012), and the numerical simulations were carried out by running GPUSPH on six NVIDIA Tesla C2050 GPUs. The numerical model simulates $8 \mathrm{~s}$ of the physical experiment, and it requires approximately $70 \mathrm{~h}$ of computation when the finest particle size of $0.005 \mathrm{~m}$ is used.

\section{Tsunami on Type I bridge}

\subsection{Free-surface evolution}

Comparison of the time-series free-surface elevations at two wave gages between the laboratory measurements and the numerical results for the tsunami impacting Type I bridge is shown in Fig. 3. Laboratory measurements at both gages show that the generated tsunami is in the form of a bore with a train of free-surface undulations. In general, the numerical model also predicts a similar bore profile with all particle sizes at both gages. When looking into the detailed comparison in terms of wave arrival prediction, the arrival of the tsunami predicted by GPUSPH matches well with the experimental data for all three particle sizes at Gage 1. It is seen that the numerical result with $\Delta p=0.01 \mathrm{~m}$ captures the first peak wave at Gage 1, but simulations with the other two particle sizes give under-predictions with a wave height difference within 1-2 particle sizes. As the tsunami continues to propagate toward the bridge, the wavefront of the tsunami reaches Gage 2 that is $1.5 \mathrm{~m}$ shoreward of Gage 1, the predicted bore slope is slightly steeper than the one in the laboratory. The numerical simulation with $\Delta p=0.005 \mathrm{~m}$ captures the first bore peak, but the sharp free-surface change indicates

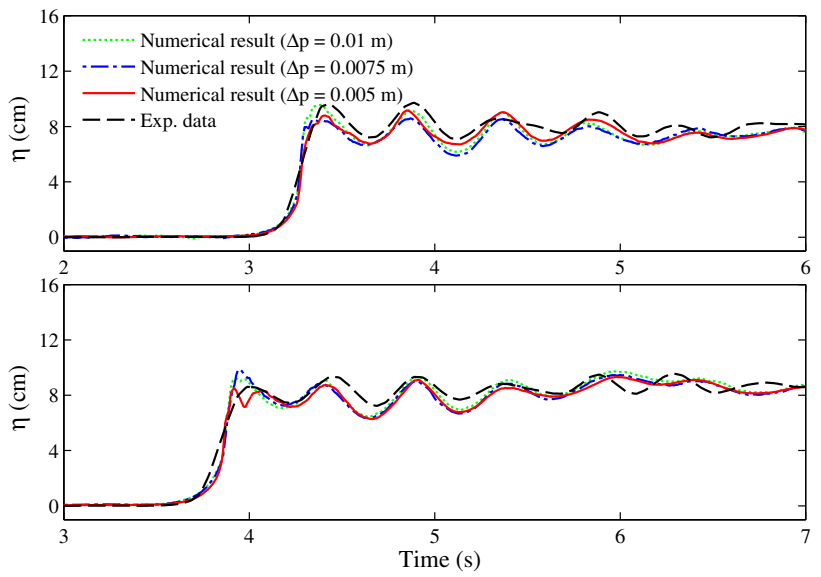

Fig. 3 Comparison of the measured and simulated free-surface profiles at Gage 1 (top panel) and Gage 2 (bottom panel) of Type I bridge test

that the predicted tsunami front with $\Delta p=0.005 \mathrm{~m}$ breaks somewhere between Gages 1 and 2. On the other hand, overpredictions are given when particle sizes $\Delta p=0.0075$ and $0.01 \mathrm{~m}$ are used, because they are too coarse to resolve the first free-surface undulation. With respect to the prediction on free-surface undulations, GPUSPH correctly simulates the number of surface waves and the wave evolution process with all three particle sizes, but there are slight phase differences, and free-surface elevation differences (within the range of a few particle sizes) with the laboratory measurements, which are attributed to the fact that the tsunami generation in our numerical model is different from the one used in the laboratory. Overall, it could be concluded that the alternative tsunami generation method based on Eq. (9) that avoids the tedious trial-and-error approach for reproducing the laboratory gate falling process is effective, and the agreement with the experimental data is acceptable for further analysis.

Next, the tsunami impingement on Type I bridge that consists of a single deck and a pier predicted by GPUSPH with a particle size of $\Delta p=0.005 \mathrm{~m}$ is shown in Fig. 4. The free-surface snapshots predicted by GPUSPH were obtained by using the free-surface detection method presented in Wei et al. (2016). Figure 4a shows the snapshot of free-surface profile at $t=4.34 \mathrm{~s}$ when the tsunami arrives at the front of the bridge, it is seen that the tsunami is unsteady and turbulent as the wavefront is not smooth. Due to the fast propagation speed, the tsunami already impinged on the bridge only after $0.3 \mathrm{~s}$ as shown in Fig. 4b. It is seen underneath the bridge that the tsunami front almost reaches the nearshore side of the bridge, and above the bridge there are a scatter of water drops splashed into the air due to the tsunami-bridge impact. Moreover, a surface undulation is approaching the bridge offshore. Figure $4 \mathrm{c}$ shows the tsunami profile at $t=4.94 \mathrm{~s}$ that is $0.3 \mathrm{~s}$ after the previous free-surface profile presented in Fig. 4b. Although the tsunami front passes the bridge, it is 
Fig. 4 Snapshots of a tsunami impinging on Type I bridge with both onshore (left panel) and offshore (right panel) views. a $t=4.34 \mathrm{~s}, \mathbf{b} t=4.64 \mathrm{~s}$, c $t=4.94 \mathrm{~s}$, and $\mathbf{d} t=5.24 \mathrm{~s}$. The color denotes cross-shore velocity
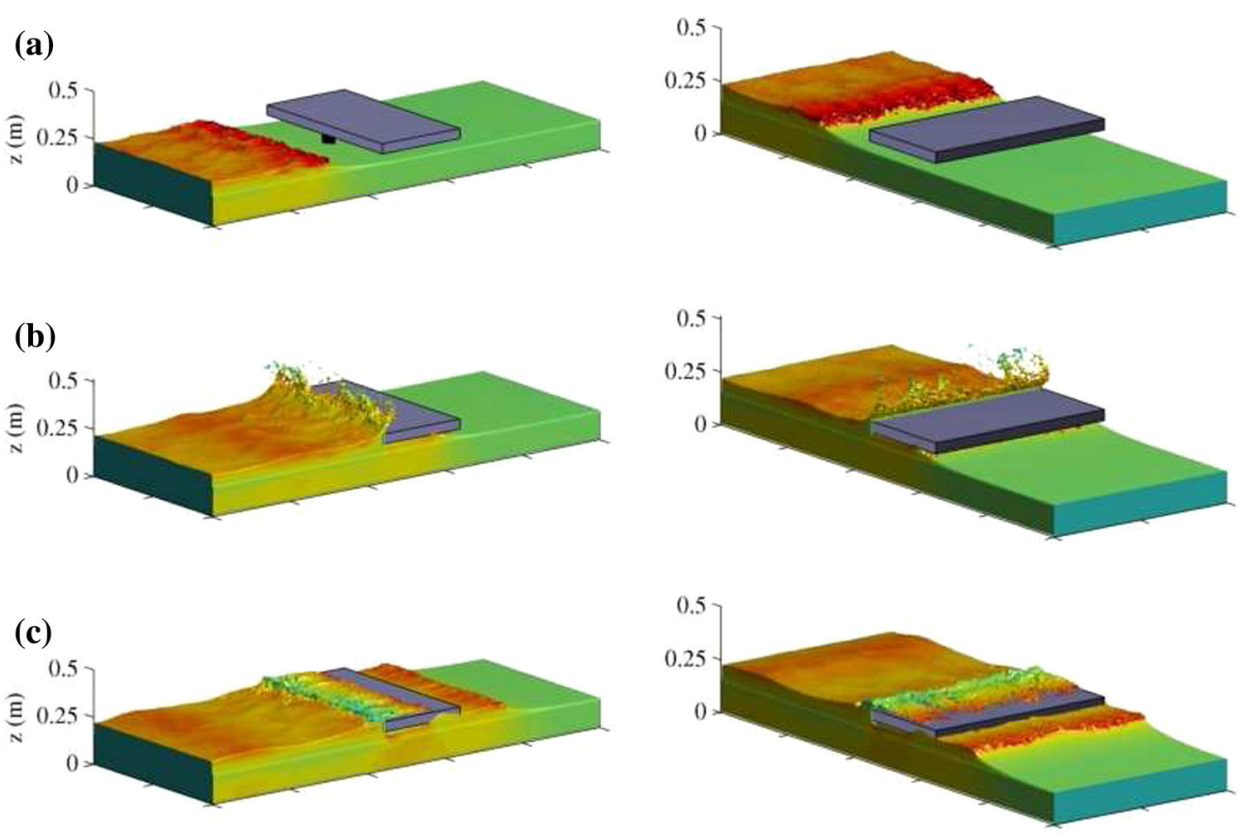

(d)

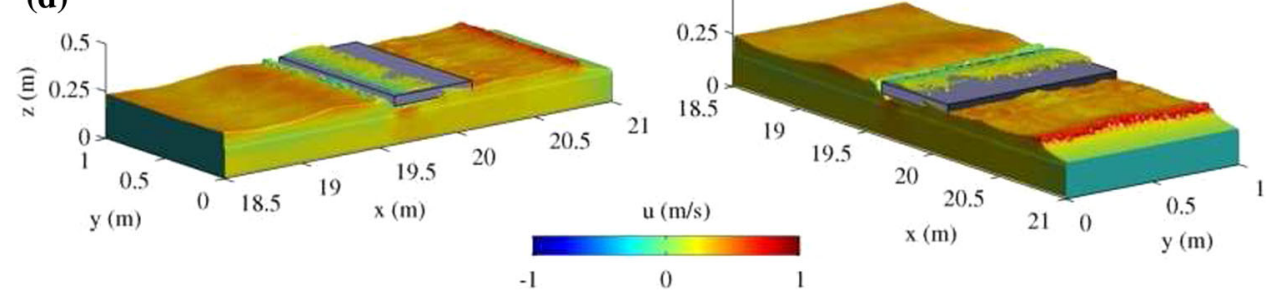

slowed down due to the interaction with the bridge, and this is confirmed by the fact that the tsunami propagation distance from $t=4.64$ to $4.94 \mathrm{~s}$ is shorter than the one from $t=4.34$ to $4.64 \mathrm{~s}$, as indicated in the aforementioned snapshots. Furthermore, some water is accumulated above the offshore side of the bridge, and that is due to: (1) the falling of the wave drops previously splashed into the air as seen in Fig. $4 \mathrm{~b}$, and (2) the wave overtopping due to the incoming surface undulation observed earlier in Fig. 4b. Finally, it is seen that another surface undulation reaches the front of the bridge, and the tsunami front continues to propagate shoreward in Fig. $4 \mathrm{~d}$ at $t=5.24 \mathrm{~s}$.

\subsection{Hydrodynamic forces}

The time-series tsunami horizontal and vertical forces on Type I bridge superstructure (i.e., the single deck) is shown in Fig. 5. For the horizontal force, the tsunami arrival time and the force increasing process are correctly predicted by the model with three particle sizes. However, discrepancies between the experimental data and the numerical simulation are observed for the horizontal peak force. Simulations with a coarse particle size such as $\Delta p=0.0075$ and $0.01 \mathrm{~m}$ are able

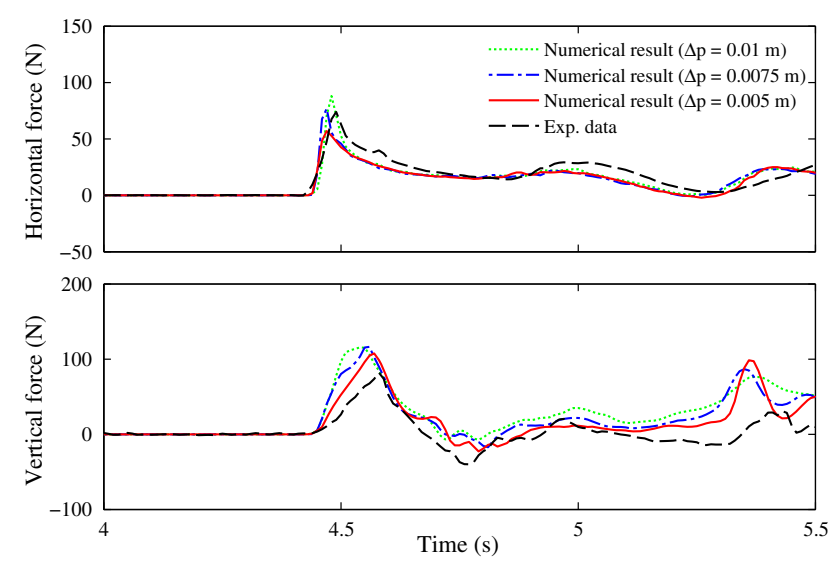

Fig. 5 Comparison of the measured and simulated hydrodynamic forces on Type I bridge. Horizontal force (top panel), and vertical force (bottom panel)

to capture the force peak, but numerical under-prediction is observed with the particle size of $\Delta p=0.005 \mathrm{~m}$, and this is due to the fact that the predicted tsunami front with this particle size already broke (see the free-surface profile at Gage 2 in Fig. 3) before the arrival at the front of the bridge, resulting in a less strong impact on the deck. After $t=4.5 \mathrm{~s}$, 
it is seen that the predicted horizontal forces with all three particle sizes follow the same trend, indicating the numerical convergence of the horizontal force prediction by GPUSPH. Due to the slight phase difference between the numerical and laboratory tsunamis (see Fig. 3), there is a similar pattern in the horizontal force comparison. For the vertical force, the numerical model also predicts the correct arrival time, but force over-predictions are seen for all three particle sizes. These differences may be caused by: (1) the air might be compressed and it serves as a buffer zone when the water hits the bottom of the deck in real life; but (2) the current version of GPUSPH is based on a single-phase assumption and unable to consider the air-phase in the model, resulting in the above over-prediction (Mokos et al. 2015). After the peak force at $t=4.6 \mathrm{~s}$, the model captures the reduction of the vertical force as measured in the laboratory. Furthermore, a good prediction is obtained by the model as the tsunami passes the bridge around $t=5 \mathrm{~s}$. Similar to the horizontal force comparison, the phase difference in the undulation also influences the numerical prediction on the vertical force, as can be seen around $t=5.4 \mathrm{~s}$. In summary, GPUSPH is able to predict the overall trend of the hydrodynamic force when a tsunami impacts Type I bridge, but the alternative tsunami generation method and the single-phase assumption in the numerical model bring about some discrepancies.

\section{Tsunami on Type II bridge}

\subsection{Free-surface evolution}

Comparison of the time-series free-surface elevations at two wave gages between the laboratory measurements and the numerical results for the tsunami impacting Type II bridge is shown in Fig. 6. It is worth pointing out that in the laboratory both the initial water depth and the target tsunami wave height of Type II bridge test were the same with those of Type I bridge test (Nakao et al. 2013). However, the measured timeseries free-surface profiles at Gage 1 in Fig. 6 are obviously different from those measured at the same location as shown in Fig. 3. This difference confirms that a tsunami generated by the gate falling method in Nakao et al. (2013) could not even be well reproduced in the laboratory, and therefore an alternative tsunami generation method based on the actual measured experimental data is necessary when the laboratory experiment is simulated by a numerical model. In general, the free-surface profile comparison between the laboratory measurement and the numerical simulation for Type II bridge is very similar to the one in Type I bridge, as shown in Fig. 3. For example, the arrival of the tsunami is well simulated by the numerical model; the steep tsunami front predicted by GPUSPH also broke before it reaches Gage 2 with a fine particle size of $\Delta p=0.005 \mathrm{~m}$. Furthermore, the particle
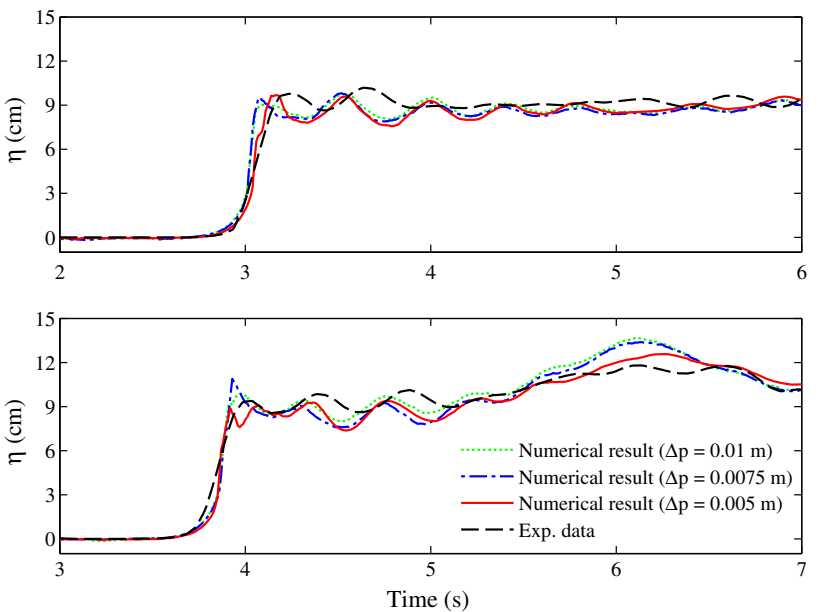

Fig. 6 Comparison of the measured and simulated free-surface profiles at Gage 1 (top panel) and Gage 2 (bottom panel) of Type II bridge test

size of $\Delta p=0.005 \mathrm{~m}$ is able to resolve the wave reflection from the bridge around $t=6 \mathrm{~s}$ as observed at Gage 2 .

Next, the tsunami impingement on Type II bridge that consists of a deck, four girders, and a pier predicted by GPUSPH with the particle size of $\Delta p=0.005 \mathrm{~m}$ is shown in Fig. 7 . Figure $7 \mathrm{a}$ shows that the tsunami approaching the front of the bridge at $t=4.31 \mathrm{~s}$, and its wavefront is similar to that observed in Type I bridge test (Fig. 4a). Then Fig. 7b shows that after $0.4 \mathrm{~s}$ the tsunami impacts the bridge deck and the offshore girder, resulting in considerable amount of water splashed in the air. Furthermore, the tsunami front reaches the nearshore side of the bridge, but some water is trapped between girders. Meanwhile, a surface undulation is propagating toward the bridge offshore. Figure 7c shows that the surface undulation observed in Fig. $7 \mathrm{~b}$ breaks in front of the bridge at $t=5.01 \mathrm{~s}$, and the flow is accumulated in front of the bridge due to the blockage effect of girders. It is seen that the main flow under girders is positive based on the color bar at the bottom of Fig. 7. However, the velocity field between girders is actually negative, indicating that flow rotations are formed under the bridge. Finally, Fig. 7d shows at $t=5.31 \mathrm{~s}$ that the tsunami front passes the bridge, and the flow pattern under the offshore girder is similar to a hydraulic jump under a sluice gate in an open channel.

\subsection{Hydrodynamic forces}

The time-series tsunami horizontal and vertical forces on Type II bridge superstructure (i.e., the deck and four girders) is shown in Fig. 8. For the horizontal force, the tsunami arrival time is correctly predicted by the model with three particle sizes. GPUSPH with the particle size of $\Delta p=0.005 \mathrm{~m}$ is able to capture the force increasing process and the peak force well, but over-predictions are given by the model when particle sizes $\Delta p=0.0075$ and $0.01 \mathrm{~m}$ are used, and this 
Fig. 7 Snapshots of a tsunami bore impinging on Type II bridge with both onshore (left panel) and offshore (right panel) views. a $t=4.31 \mathrm{~s}, \mathbf{b}$ $t=4.71 \mathrm{~s}, \mathbf{c} t=5.01 \mathrm{~s}$, and $\mathbf{d}$ $t=5.31 \mathrm{~s}$. The color denotes cross-shore velocity (a)

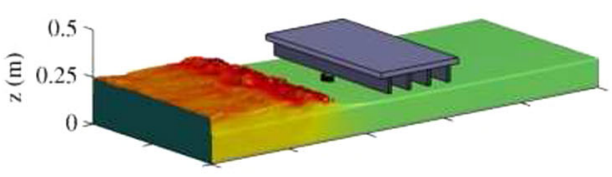

(b)
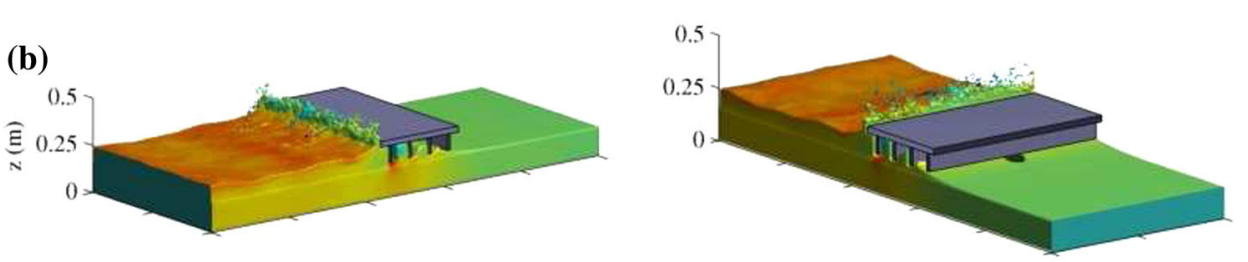

(c)
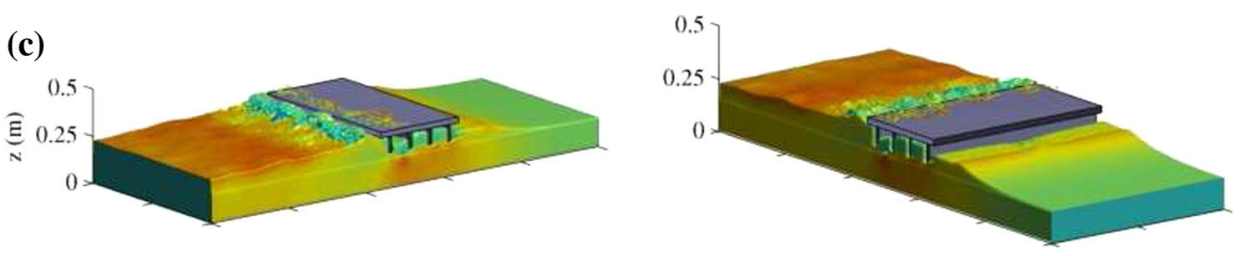

(d)
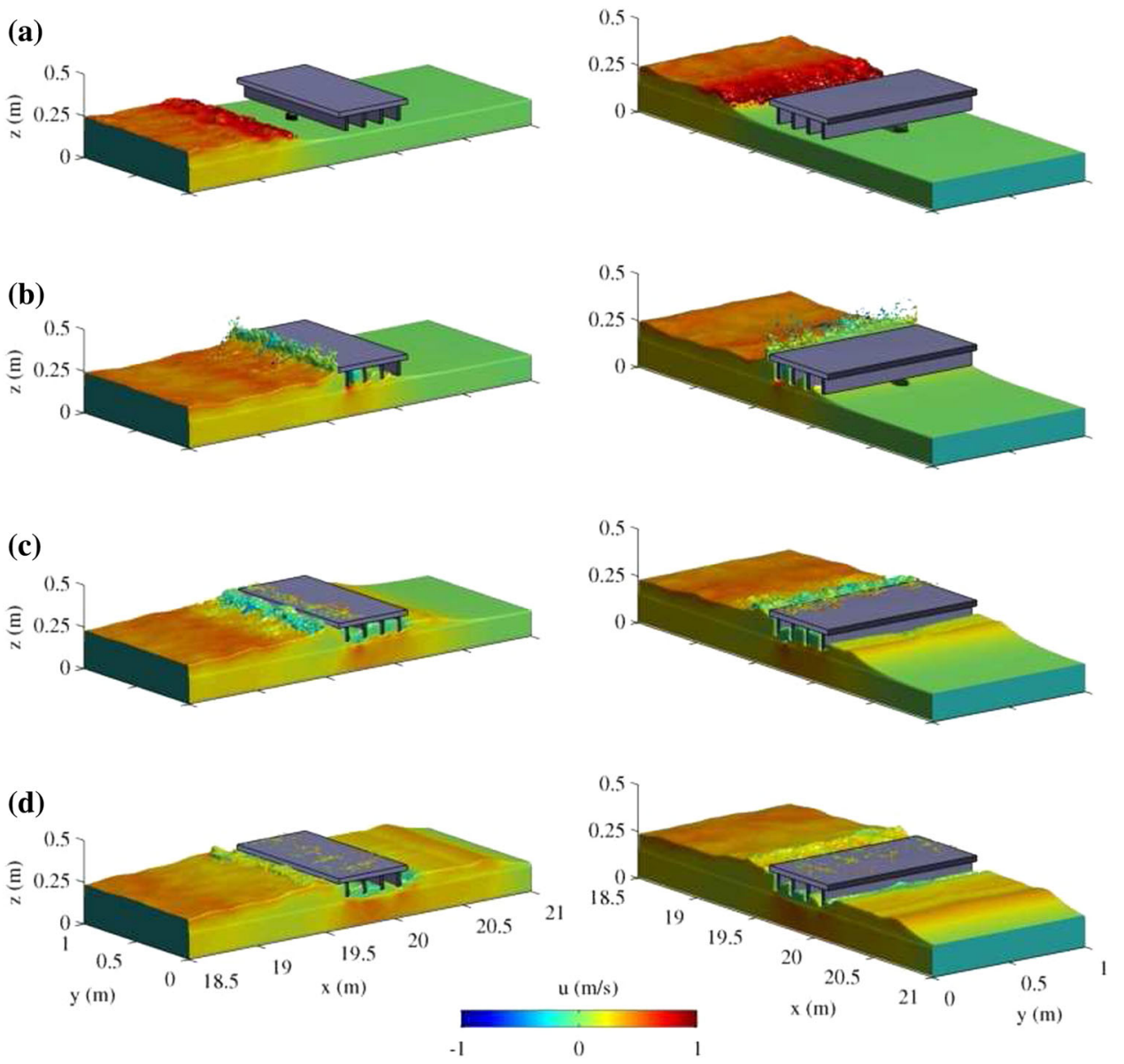
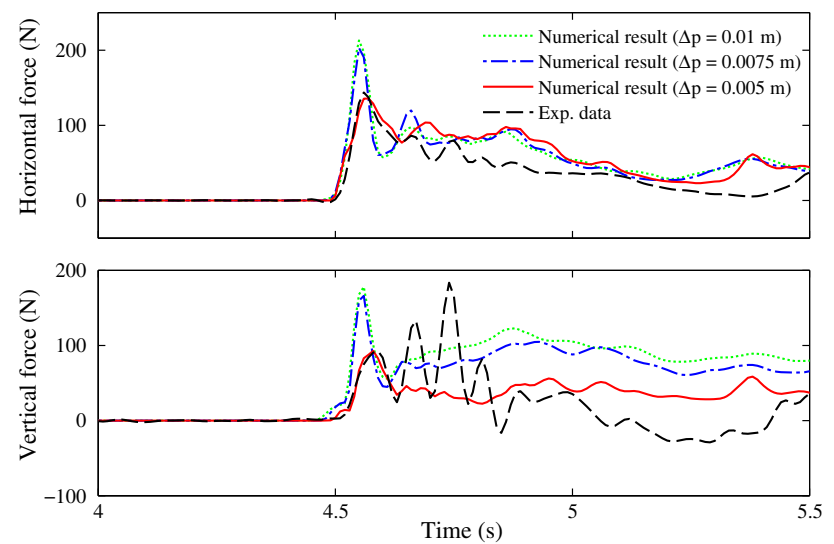

Fig. 8 Comparison of the measured and simulated hydrodynamic forces on Type II bridge. Horizontal force (top panel), and vertical force (bottom panel)

is due to that the two particle sizes are too coarse to well represent the girder dimension in the cross-shore direction (i.e., $0.02 \mathrm{~m}$ ). It is also seen that the numerical model also correctly predicts the retreat of the horizontal force with $\Delta p=0.005 \mathrm{~m}$, and discrepancies can be observed starting around $t=4.6 \mathrm{~s}$ due to the slight phase difference between the numerical and laboratory tsunamis (see Fig. 6).
Similar to the numerical prediction in Type I bridge test, the predicted horizontal forces with all three particle sizes follow the same trend after the tsunami front passes the bridge around $t=4.7 \mathrm{~s}$, also indicating the numerical convergence of the horizontal force prediction. For the vertical force, the numerical model also predicts the correct arrival time, but over-predictions of the first force peak are seen for the two coarse particle sizes, as seen earlier in the horizontal force comparison. The numerical model with the particle size of $\Delta p=0.005 \mathrm{~m}$ accurately predicts the full profile of the first peak of the vertical force. In the laboratory measurement, there are several force peaks that are even larger than the first one when the tsunami impacts the bridge between $t=4.6$ and $4.8 \mathrm{~s}$. However, the numerical simulation simply misses all these force peaks. Possible explanations for this discrepancy are: (1) there was a possible vibration of the laboratory bridge caused by the wave impact, but the numerical bridge model is fixed and therefore can not consider the dynamic response of the bridge; and (2) the single-phase model does not take the air into account, and it already gave under-predictions to free-surface undulations with a few particle sizes at Gage 2 (see the result between $t=4.2$ and $5.2 \mathrm{~s}$ in Fig. 6). The difference is further observed for the remaining part of vertical force comparison, and again it is caused 
by the different tsunami wave generation method used by the numerical model. However, it should be emphasized that the numerical result with the particle size of $\Delta p=0.005 \mathrm{~m}$ matches closer to the experimental data than those simulated with coarse particle sizes. Therefore, the following section only utilizes the particle size of $\Delta p=0.005 \mathrm{~m}$ to analyze tsunami mitigation on bridges.

\section{Tsunami forcing mitigation}

In the previous two sections, the capability of GPUSPH to predict tsunami forces on bridges was evaluated by simulating the laboratory experiment of Nakao et al. (2013). Although discrepancies were observed in numerical simulations because a different tsunami generation method has to be used in the single-phase numerical model, GPUSPH with a fine particle size of $0.005 \mathrm{~m}$ is able to reasonably predict the first peak of tsunami forces on bridges, which are generally large, and therefore critical for the bridge safety design. In this section, additional numerical experiments based on those presented in previous sections are carried out to examine the efficiency of tsunami mitigation on bridges by two types of man-made structures, i.e., a service road bridge and a breakwater. Although two types of bridges of Nakao et al. (2013) were considered earlier in this work, the following analysis on tsunami force mitigation is only conducted for Type II bridge that has a deck and four girders, because it resembles better a real-life bridge than Type I bridge does. The major activities/steps include: (1) adding a structure offshore of Type II bridge (the bridge hereafter); (2) rerun the tsunami impacting a bridge test as presented in Sect. 5, and (3) comparing the tsunami peak force reduction on the bridge and analyzing the hydrodynamic force on the added structures (i.e., the service road bridge and the breakwater). It should be pointed out that since the followings are pure numerical experiments, it is consistent to analyze the force reduction on the basis of the numerical force computed by GPUSPH in Sect. 5.

\subsection{Tsunami mitigation by a service road bridge}

A service road bridge is a local bridge running parallel to a higher-speed, limited-access highway bridge. During the 2011 Great East Japan Tsunami, superstructures in 12 bridges including service road for pedestrian on national highway route 45 of Japan (main route along the Pacific coast of Tohoku area) were washed away (Hoshikuma and Zhang 2013). In the laboratory work of Nakao et al. (2013), the effect of a service road bridge on tsunami force reduction on bridge superstructures was also investigated. However, their laboratory data and setup are not available to the authors for model-data comparison. Therefore, numerical experiments are conducted to investigate tsunami mitigation by a service road bridge in this study.

The sketch of numerical experiments on tsunami mitigation by a service road bridge is shown in Fig. 9. Two types of service road bridges, denoted as I and II hereafter, are considered. For Type I service road bridge (see Fig. 9a, b), it is inspired by the setup of Type I bridge of Nakao et al. (2013) (see Fig. 2c, d), and the tsunami force on it predicted by GPUSPH was presented in Sect. 4. The length of Type I service road bridge in the cross-shore direction is $0.125 \mathrm{~m}$, and the clearance between the service road bridge and the main bridge is set to be $0.05 \mathrm{~m}$ by following Nakao et al. (2013). Similarly, Type II service road bridge follows the setup of Type II bridge of Nakao et al. (2013) (see Fig. 2e, f), and the tsunami force on it predicted by GPUSPH was presented in Sect. 5. However, the considered Type II service road bridge only has two girders, and the cross-shore dimension and the clearance with the main bridge are the same with those of Type I service road bridge.

Figure 10 shows the difference of tsunami impacting a bridge with and without a service road bridge at $t=4.71 \mathrm{~s}$. The tsunami impacting the bridge without any protection is first presented in Fig. 10a, and it is the same snapshot shown in Fig. 7b. By adding Type I service road bridge, the tsunami impact has been greatly reduced in terms of wave splash and overtopping, as indicated in Fig. 10b. Furthermore, Type II service road bridge also significantly reduces the tsunami impacting the bridge, as shown in Fig. 10c. However, due to the existence of two girders, Type II service road bridge has a stronger blockage effect on the tsunami than that of Type I service road bridge.

Next, the effect of a service road bridge on tsunami force reduction is presented in Fig. 11. As pointed out earlier that the tsunami force on a bridge without protection is the numerical result of GPUSPH presented in Fig. 8. It is seen that with the Type I service road bridge there are about 19 and $16 \%$ tsunami peak forces could be reduced in the horizontal and vertical directions, respectively. Furthermore, the tsunami force on the service road bridge itself is not significant because it is made of a single deck. On the other hand, Type II service road bridge with two girders is able to greatly reduce the horizontal and vertical peak forces up to 50 and $36 \%$, respectively. However, the tsunami force on Type II service road bridge is almost equal to the one on the bridge without any protection, and therefore the service road bridge may not survive the tsunami.

\subsection{Tsunami mitigation by a breakwater}

A breakwater is a structure built to reduce the offshore wave impact on harbor and nearshore structures. There is a long history in Japan of using breakwaters for mitigating a tsunami disaster (see e.g., Fukuuchi and Ito (1966); Fujima (2006)). 


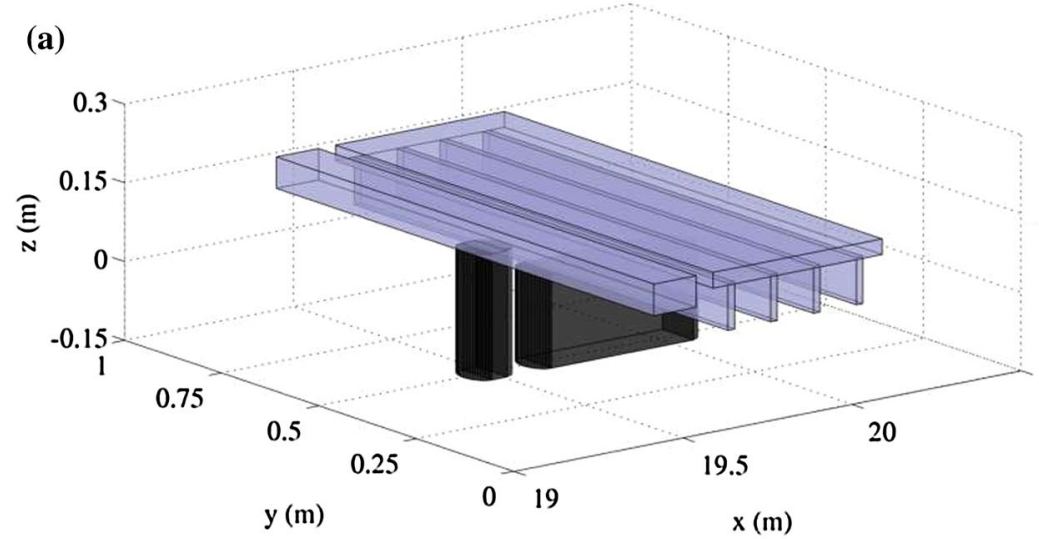

(b)
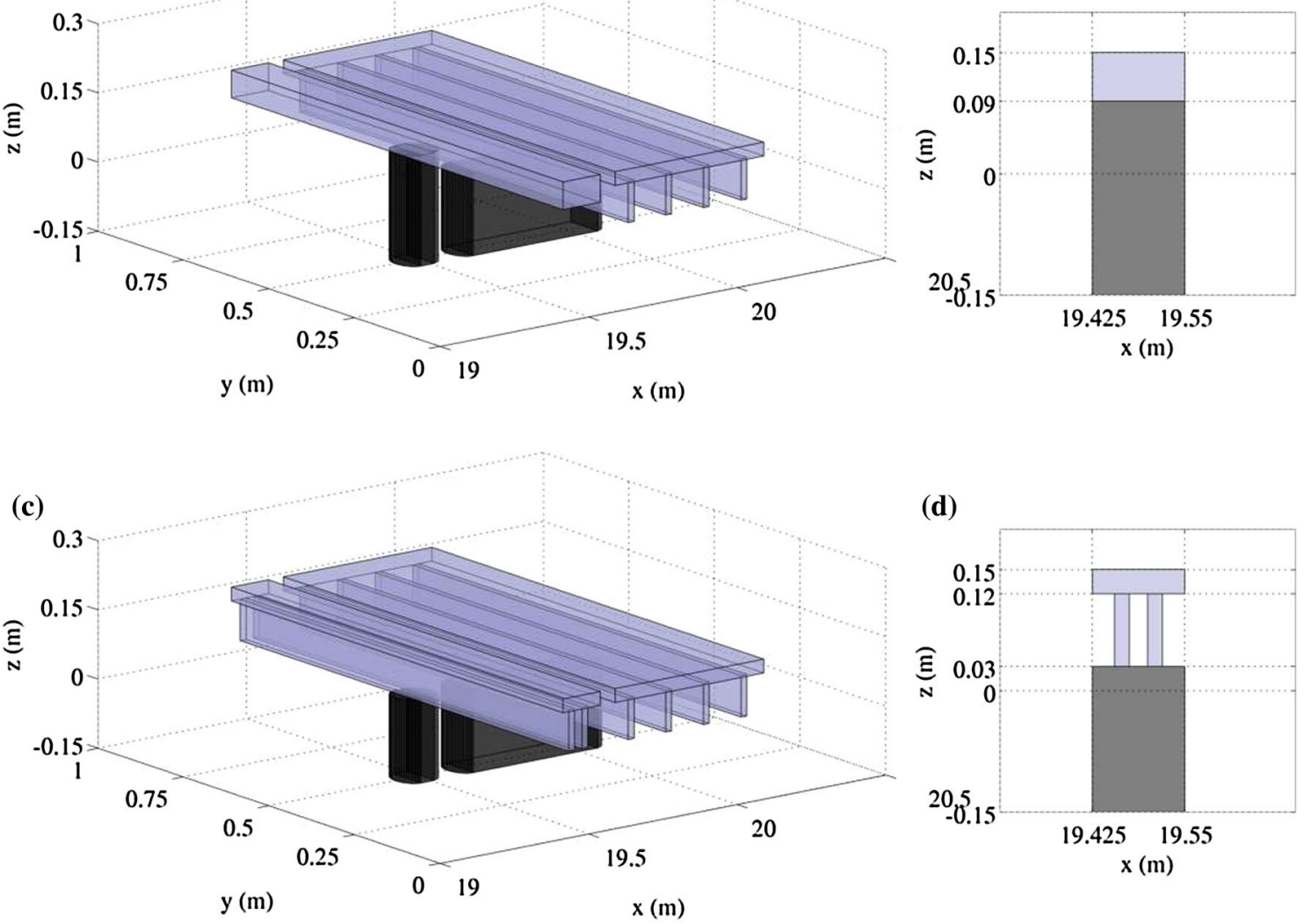

(d)

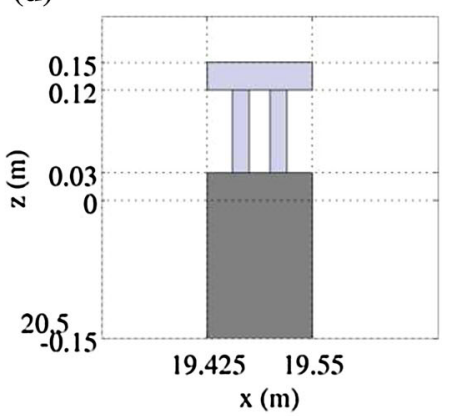

Fig. 9 Sketch of the numerical experiment setup on tsunami mitigation by a service road bridge. a $3 D$ view of Type I service road bridge, b side view of Type I service road bridge, $\mathbf{c} 3 D$ view of Type II service road bridge, and $\mathbf{d}$ side view of Type II service road bridge

Fig. 10 Tsunami mitigation on wave impacting a bridge with and without a service road bridge at $t=4.71 \mathrm{~s}$. Onshore view (left panel) and offshore view (right panel). a A bridge without protection (the same one as Fig. 7b), b a bridge with Type I service road bridge, and c a bridge with Type II service road bridge. The color denotes cross-shore velocity (a)
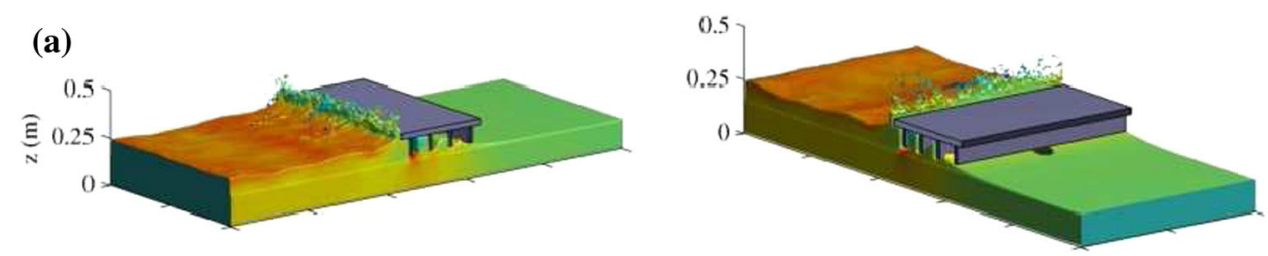

(b)
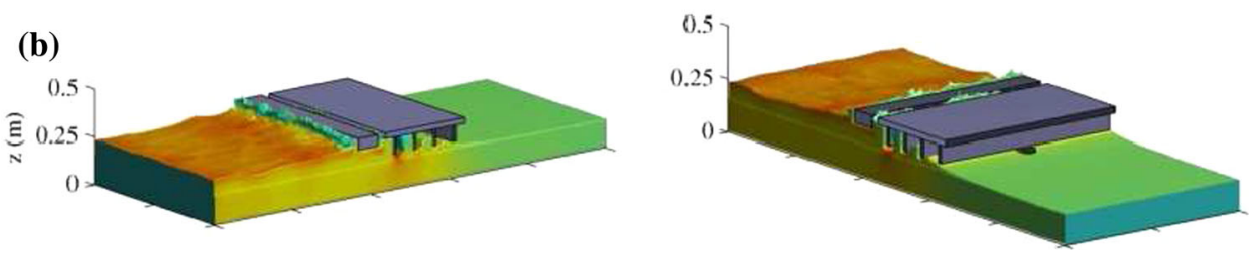

(c)

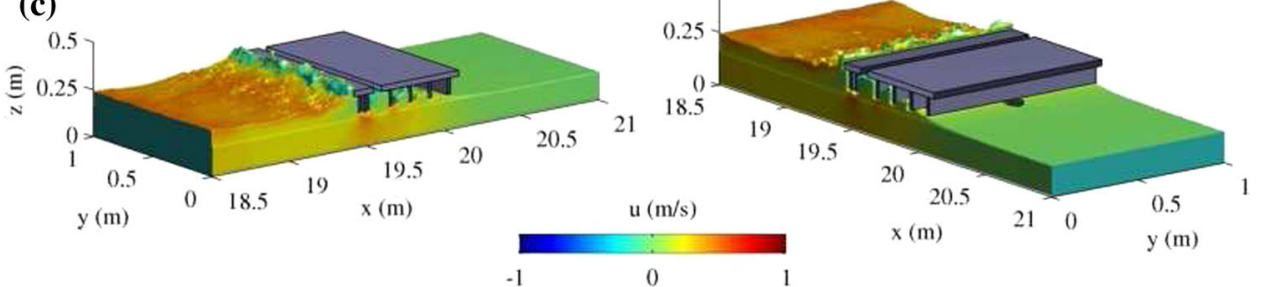


Fig. 11 Tsunami mitigation on hydrodynamic forces on a bridge by a service road bridge. a Horizontal force mitigation with Type I service road bridge, b vertical force mitigation with Type I service road bridge, $\mathbf{c}$ horizontal force mitigation with Type II service road bridge, and d vertical force mitigation with Type II service road bridge
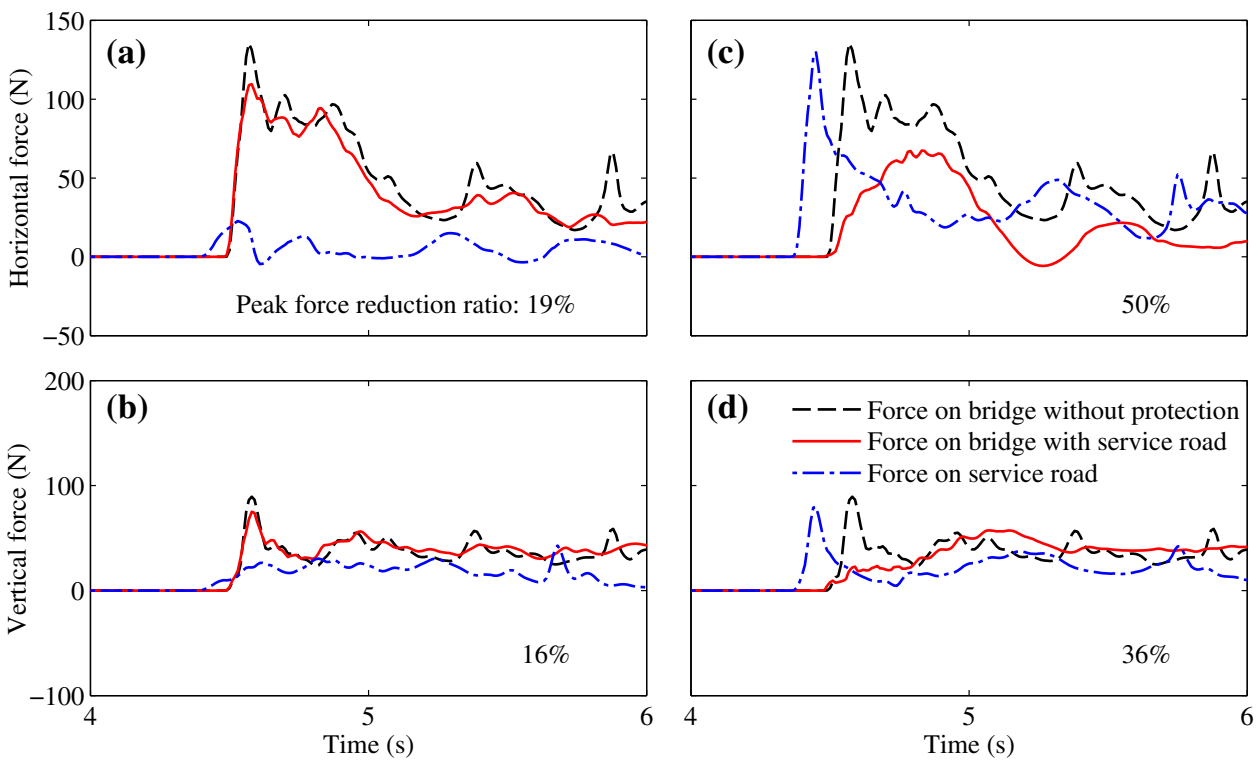

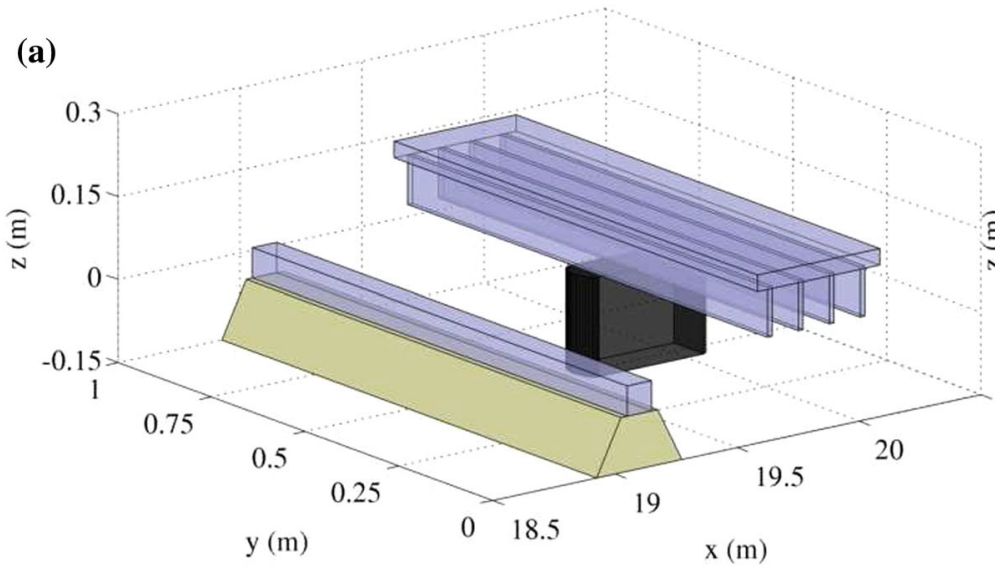

(b)

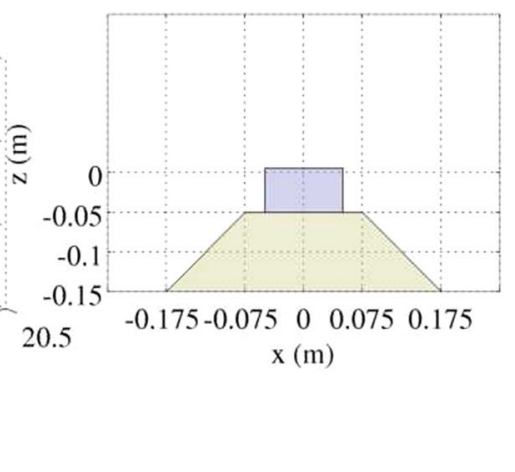

Fig. 12 Sketch of the numerical breakwater experiment setup. a $3 D$ view of the breakwater that consists of a rubble mound and a caisson, and $\mathbf{b}$ side view of the breakwater with a local coordinate system

Although it is difficult to collect data and quantify the effect of a breakwater for mitigating a tsunami impacting bridges in real life, laboratory experiment shows that breakwaters placed in front of a bridge are able to reduce tsunami force on a bridge (Iemura et al. 2007). In this study, numerical experiments are conducted to investigate the effect of a breakwater on reducing tsunami force on coastal bridges, in particular, a proper distance between a breakwater and a bridge to achieve a favorable effect.

This study considers a simplified vertical type breakwater that consists of a mound and a vertical wall (or a caisson) (Takahashi 2002), as shown in Fig. 12. It should be pointed out that the considered setup is for coastal bridges with an offshore breakwater, and it not applicable to a riverine bridge, as the breakwater would block the navigation channel. The dimension of the breakwater (e.g., the slope of the mound and the relative dimension between the mound and the cais- son) is determined by following the work of Arikawa et al. (2012). Furthermore, due to the limited width of the flume, an opening between breakwaters is not considered in this work. The primary concern of this study is to examine the influence of the distance $(D)$ between the breakwater and the bridge on reducing tsunami forces on the bridge, and the distance is measured from the center of the breakwater (i.e., the local coordinate $x=0$ in Fig. 12b) to the offshore edge of the bridge pier.

Figure 13 shows the free-surface profile difference on tsunami impacting a bridge with and without a breakwater at $t=4.71 \mathrm{~s}$. Similar to the service road bridge test, the tsunami impacts a bridge without any protection (i.e., Fig. 13a) is also the same snapshot presented in Fig. 7b. With a breakwater, the strong tsunami breaks over it in a form similar to a hydraulic jump observed in an open channel flow. It is also seen that the tsunami impacting the bridge is significantly reduced by 
Fig. 13 Tsunami mitigation on wave impacting a bridge with and without a breakwater at $t=4.71 \mathrm{~s}$. Onshore view (left panel) and offshore view (right panel). a A bridge without protection (the same one as Fig. 7b), b a bridge with a breakwater offshore $(D=0.5$ $\mathrm{m})$, c a bridge with a breakwater offshore $(D=1 \mathrm{~m})$, and $\mathbf{d}$ a bridge with a breakwater offshore $(D=1.5 \mathrm{~m})$. The color denotes cross-shore velocity
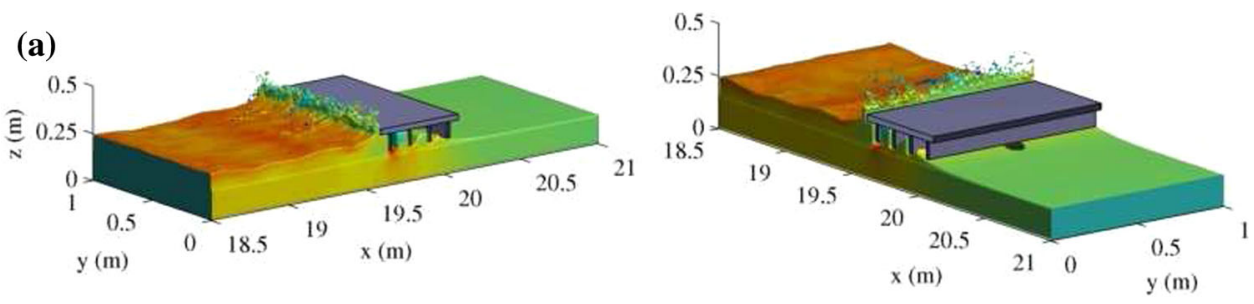

(b)
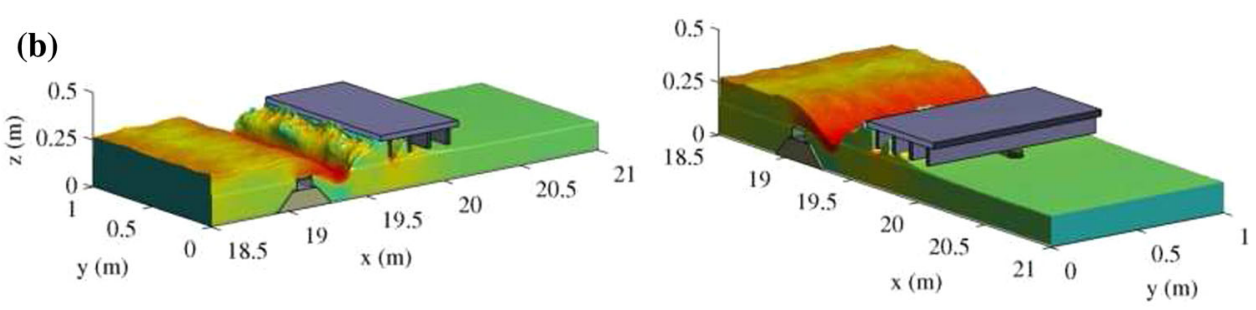

(c)
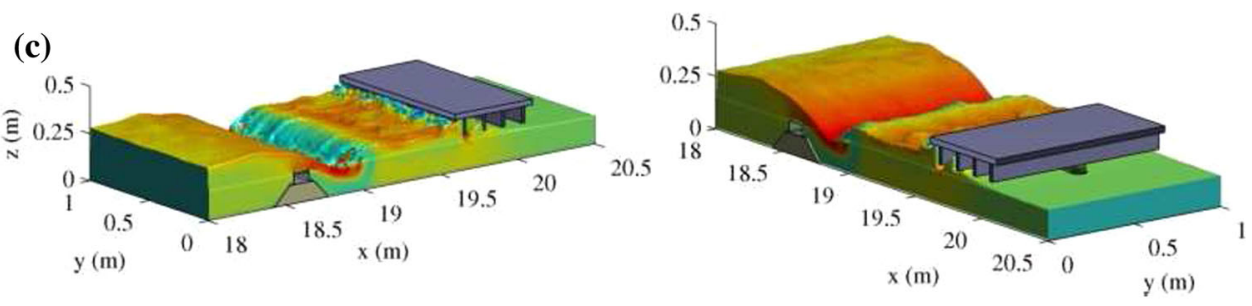

(d)

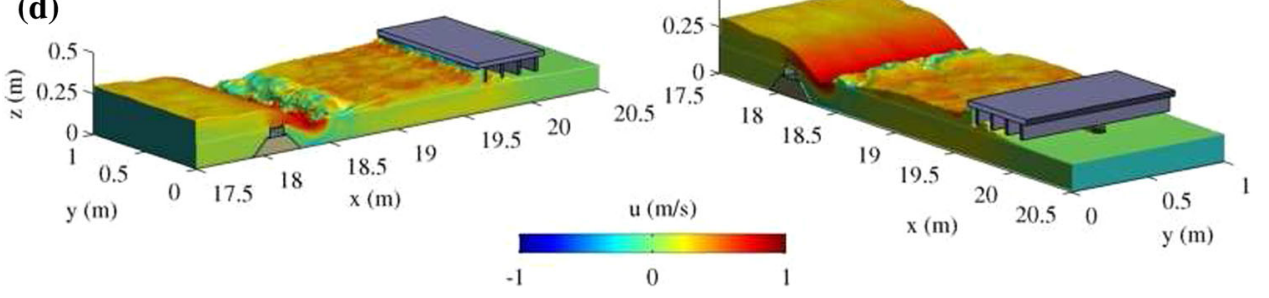

the breakwater with three different distances (i.e., 0.5, 1.0, and $1.5 \mathrm{~m}$ ), and the larger the distance among these tests, the less wave impacting the bridge.

Next, Fig. 14 shows that both horizontal and vertical forces on the bridge can be reduced by using a breakwater with distances $D=0.5,1.25$ and $2.0 \mathrm{~m}$, and it is also seen that more force is reduced when the distance is larger. However, a breakwater with a distance $D=1.25 \mathrm{~m}$ that is closer to the bridge than that with a distance $D=2$ m gives an even larger peak force reduction. Then several other distances are further tested by the numerical model, and the corresponding peak force reduction ratios are presented in Fig. 15. For this specific experimental setup (i.e., the local water depth is $0.15 \mathrm{~m}$, and the target tsunami height is $0.1 \mathrm{~m}$ ), the result shows that by increasing the distance between the breakwater and the bridge, tsunami peak forces on the bridge are further reduced, and an optimal distance for the best reduction efficiency (i.e., 50 and $58 \%$ reductions for the horizontal and vertical peak forces, respectively) is around $D=1.25 \mathrm{~m}$, which is about eight times of the local water depth, and 13

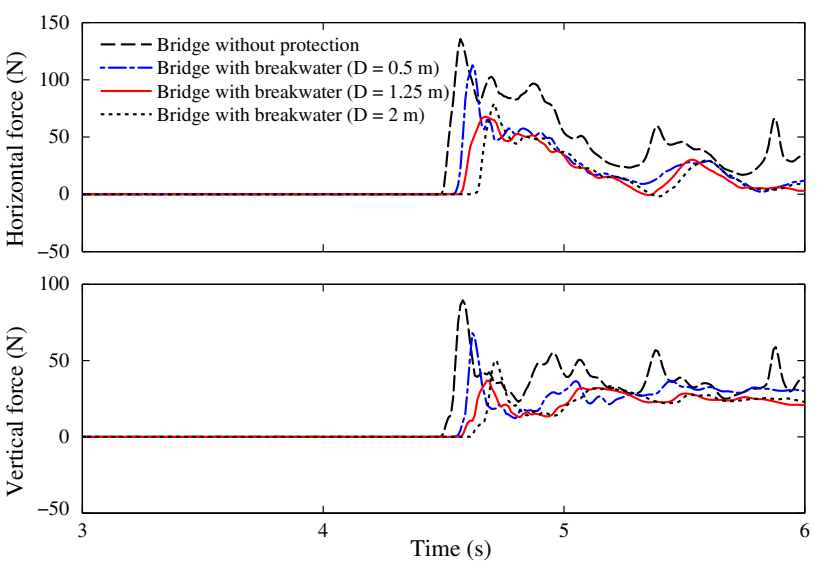

Fig. 14 Tsunami mitigation on hydrodynamic forces on a bridge by a breakwater with different distances $(D)$ to the bridge. Horizontal force (top panel), and vertical force (bottom panel)

times of the target tsunami height. It is also observed that after increasing the distance to a certain value (e.g., $1.5 \mathrm{~m}$ ), there is no further gain regarding the peak force reduction, 


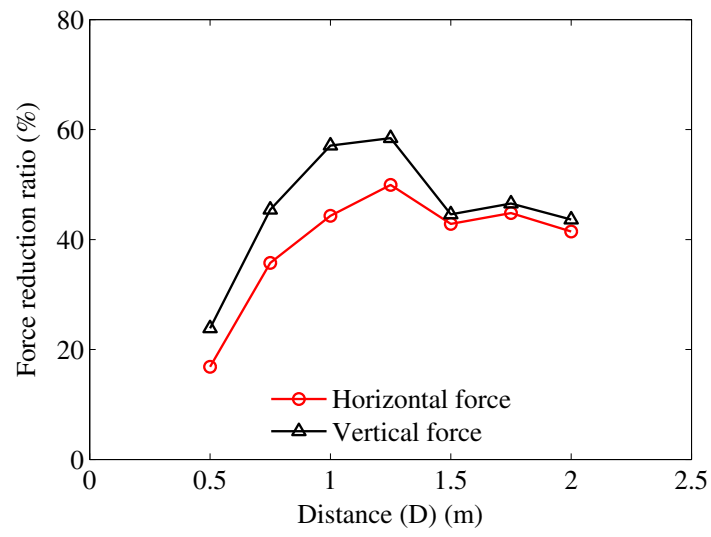

Fig. 15 The reduction ratio of tsunami forces on a bridge in terms of the distance $(D)$ between the breakwater and the bridge
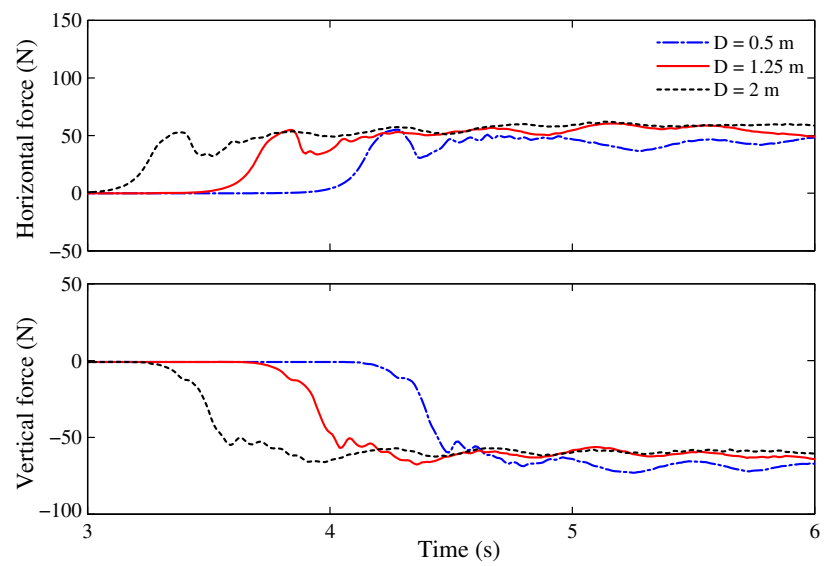

Fig. 16 Hydrodynamic forces on the breakwater with different distances $(D)$ to the bridge. Horizontal force (top panel), and vertical force (bottom panel)

but they are still more efficient than a short distance such as $0.5 \mathrm{~m}$.

There is no doubt that a breakwater can reduce tsunami forces on a bridge as demonstrated above, but in reality breakwaters were actually damaged by a tsunami (see e.g., Imamura and Anawat 2011). Figure 16 shows the tsunami force on the breakwater caisson. It is seen that although the arrival time is different among breakwaters with different distances to the bridge, the forces are actually very similar among them, and these strong forces may cause the failure of the breakwater. Furthermore, Fig. 17 shows a vertical 2D velocity field around a breakwater along $y=0.5 \mathrm{~m}$ and a distance between the breakwater and the bridge $D=1 \mathrm{~m}$. The velocity field is plotted on a fixed grid, and the velocity is obtained by a procedure presented by Wei et al. (2016). Basically, the velocity information on the fixed grid is interpolated from the neighboring SPH moving particles by using an SPH averaging. It is seen that the tsunami front jet with a high velocity field is plunging upon the seafloor, and it may

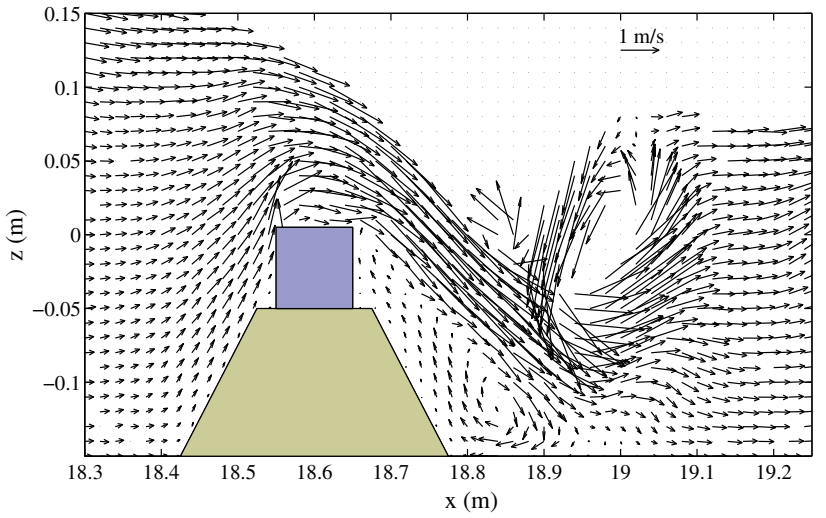

Fig. 17 The velocity field around the breakwater (the cross-section along $y=0.5 \mathrm{~m}$ ) with a distance to the bridge $D=1 \mathrm{~m}$ at $t=4.71$ s. The corresponding free-surface profile snapshot is shown in Fig. $13 \mathrm{c}$

result in a strong erosion. Moreover, the lee side slope of the breakwater may also be scoured by the rotating flow.

\section{Conclusions}

In this paper, tsunami forces on bridge superstructures, and mitigation of tsunami forces on bridges have been investigated by an SPH model, GPUSPH, which is based on the weakly compressible SPH method and implemented by utilizing the latest GPU parallel computing techniques. This study was carried out by intensively utilizing the laboratory experiment of Nakao et al. (2013), who generated a tsunami by a gate falling method and examined the tsunami impact force on different configurations of bridge superstructures. In the first part of this work, the capability of GPUSPH to predict tsunami forces on bridges was evaluated by comparing with the experimental data of Nakao et al. (2013). It has been seen that the model with a new tsunami generation method and a relatively fine particle size is able to predict the overall trend of tsunami forces on bridges, in particular, the first peak force that is essential for bridge safety design. Then in the second part of this paper, additional numerical experiments built upon the first part were carried out to examine the efficiency of tsunami mitigation by a service road bridge and an offshore breakwater. Overall, major contributions and findings in this study include:

1. This study proposes an alternative tsunami wave generation method that makes use of the laboratory free-surface measurements, and it can be adopted by other numerical models for wave generation;

2. The numerical model of GPUSPH is able to accurately predict forces on structures if the experimental setup is well-designed, and it can also capture the overall trend 
of tsunami forces on a bridge in a dynamically changing environment;

3. Numerical study in this work confirms the laboratory work finding of Nakao et al. (2013) that a service road bridge can also reduce tsunami forces on a bridge if it survives the tsunami impact, and a two-girder service road bridge is more efficient because of the blockage effect of the girders;

4. An offshore breakwater can also reduce tsunami forces on a nearshore bridge. Based on the specific setup of this study, an optimal distance between the breakwater and the bridge to achieve the best reduction effect is about eight times of the local water depth or 13 times of the incoming tsunami height. However, the breakwater may also fail because of the large tsunami loading and the strong scouring in its lee side.

Finally, it should be noted that the numerical model is based on a single-phase assumption, and it does not consider bridge structure dynamics; thus it could not predict the high-frequency tsunami force on bridges as shown in Fig. 8. But these forces should be taken into account in reality since they may escalate the bridge failure. Furthermore, floating debris from the damaged service roads and breakwaters by tsunamis could further impact the bridge (e.g., Saatcioglu et al. 2006), and they may also cause extra impact forces beyond the tsunami hydrodynamic force studied in this work. Therefore, future effort should be made to address more complex processes involving tsunami, floating debris, and bridge interaction.

Acknowledgments The authors acknowledge the support from the Office of Naval Research, Littoral Geosciences, and Optics Program. R.A.D. further acknowledges the ATHOS Consortium and its member organizations for their contributions to the GPUSPH code. The numerical simulations were carried out at the Graphics Processing Lab Cluster of Johns Hopkins University, which is sponsored by the National Science Foundation Grant MRI-0923018.

\section{References}

Arikawa T, Sato M, Shimosako K, Hasegawa I, Yeom GS, Tomita T (2012) Failure mechanism of Kamaishi breakwaters due to the Great East Japan earthquake tsunami. In: Coastal engineering proceedings, vol 1(33), structures-16

Arnason H, Petroff C, Yeh H (2009) Tsunami bore impingement onto a vertical column. J Disaster Res 4(6):391-403

Dalrymple RA, Knio O (2000) SPH modelling of water waves. In: Coastal dynamics 01, ASCE, pp 779-787

Dalrymple RA, Rogers BD (2006) Numerical modeling of water waves with the SPH method. Coast Eng 53(2):141-147

Fujima K (2006) Effect of a submerged bay-mouth breakwater on tsunami behavior analyzed by $2 \mathrm{D} / 3 \mathrm{D}$ hybrid model simulation. Nat Hazards 39(2):179-193

Fukuuchi H, Ito Y (1966) On the effect of breakwaters against tsunami. In: Coastal engineering proceedings, vol 1(10)
Gomez-Gesteira M, Rogers BD, Crespo AJC, Dalrymple RA, Narayanaswamy M, Dominguez JM (2012) SPHysicsdevelopment of a free-surface fluid solver-Part 1: theory and formulations. Comput Geosci 48:289-299

Hayatdavoodi M, Seiffert B, Ertekin RC (2014) Experiments and computations of solitary-wave forces on a coastal-bridge deck. Part II: Deck with girders. Coast Eng 88:210-228

Hérault A, Bilotta G, Dalrymple RA (2010) SPH on GPU with CUDA. J Hydraulic Res 48(S1):74-79

Hoshikuma J, Zhang G (2013) Performance of seismic retrofitted highway bridges based on observation of damage due to the 2011 Great East Japan Earthquake. J JSCE 1(1):343-352

Iemura H, Pradono MH, Yasuda T, Tada T (2007) Experiments of tsunami force acting on bridge models. Proc JSCE Earthq Eng Sympos 29:902-911

Imamura F, Anawat S (2011) Damage due to the 2011 Tohoku earthquake tsunami and its lessons for future mitigation. In: Proceedings of the international symposium on engineering lessons learned from the 2011 Great East Japan earthquake

Kathiresan K, Rajendran N (2005) Coastal mangrove forests mitigated tsunami. Estuar Coast Shelf Sci 65(3):601-606

Kawashima K, Buckle I (2013) Structural performance of bridges in the Tohoku-Oki earthquake. Earthq Spectra 29(S1):S315-S338

Kosa K, Nii S, Miyahara K, Shoji M (2010) Experimental study for estimating tsunami forces acting on bridge girders. In: Proceedings of the 26th US-Japan bridge engineering workshop, Public Works Research Institute, Tsukuba, pp 1-14

Lau TL, Lukkunaprasit P, Inoue S, Ohmachi T (2011) Experimental and numerical modeling of tsunami force on bridge decks. INTECH Open Access, Croatia

Lo EYM, Shao S (2002) Simulation of near-shore solitary wave mechanics by an incompressible SPH method. Appl Ocean Res 24(5):275-286

Mokos A, Rogers BD, Stansby PK, Domínguez JM (2015) Multi-phase SPH modelling of violent hydrodynamics on GPUs. Comput Phys Commun 196:304-316

Monaghan JJ (1992) Smoothed particle hydrodynamics. Annu Rev Astron Astrophys 30:543-574

Monaghan JJ (1994) Simulating free surface flows with SPH. J Comput Phys 110(2):399-406

Nakao H, Zhang G, Sumimura T, Hoshikuma J (2013) Numerical assessment of tsunami-induced effect on bridge behavior. In: Proceedings of the 29th US-Japan bridge engineering workshop

Rustico E, Bilotta G, Gallo G, Hérault A, Del Negro C (2012) Smoothed particle hydrodynamics simulations on multi-GPU systems. In: 2012 20th euromicro international conference on parallel. Distributed and network-based processing. IEEE, Garching, pp 384-391

Saatcioglu M, Ghobarah A, Nistor I (2006) Performance of structures in Indonesia during the December 2004 great Sumatra earthquake and Indian Ocean tsunami. Earthq Spectra 22(S3):295-319

Takahashi S (2002) Design of vertical breakwaters. In: Technical report, PARI (Port and Airport Research Institute)

Tanaka N, Sasaki Y, Mowjood MIM, Jinadasa KBSN, Homchuen S (2007) Coastal vegetation structures and their functions in tsunami protection: experience of the recent Indian Ocean tsunami. Landsc Ecol Eng 3(1):33-45

Thomas S, Cox D (2011) Influence of finite-length seawalls for tsunami loading on coastal structures. J Waterway Port Coast Ocean Eng 138(3):203-214

Wei Z, Jia Y (2013) A depth-integrated non-hydrostatic finite element model for wave propagation. Int J Numer Methods Fluids 73(11):976-1000

Wei Z, Jia Y (2014) Simulation of nearshore wave processes by a depthintegrated non-hydrostatic finite element model. Coast Eng 83:93107 
Wei Z, Dalrymple RA, Hérault A, Bilotta G, Rustico E, Yeh H (2015) SPH modeling of dynamic impact of tsunami bore on bridge piers. Coast Eng 104:26-42

Wei Z, Dalrymple RA, Rustico E, Hérault A, Bilotta G (2016) Simulation of nearshore tsunami breaking by smoothed particle hydrodynamics method. J Waterway Port Coast Ocean Eng. doi:10.1061/(ASCE)WW.1943-5460.0000334
Wendland H (1995) Piecewise polynomial, positive definite and compactly supported radial functions of minimal degree. Adv Comput Math 4(1):389-396 